\section{IN BRIEF}

VIRAL IMMUNITY

APOBEC-mediated editing of viral RNA.

Bishop, K. N. et al. Science 305, 645 (2004).

A flurry of recent papers have described the retroviral-restriction activity of APOBEC proteins - notably, APOBEC3F and APOBEC 3G - involving deamination of minus-strand viral cDNAs. This paper now describes that APOBEC1 can deaminate viral RNA, which extends the role of APOBEC-family members in innate resistance to viral infection to both DNA and RNA deamination. Rat APOBEC1 caused an accumulation of cytosineto-uracil changes in HIV genomic RNA, indicating that it might have a role in inducing mutations in viruses that replicate entirely through RNA. Also, because APOBEC1 is already known to deaminate a particular cytosine residue in apolipoprotein $\mathrm{B}$ mRNA, this demonstration of viral RNA editing might indicate that there are also other cellular RNA substrates for APOBEC1.

\section{T-CELL SIGNALLING}

Discs large (Dlg1) complexes in lymphocyte activation.

Xavier, R. et al. J. Cell Biol. 165, 173-178 (2004).

When a $\mathrm{T}$ cell encounters another cell presenting its cognate antigen, an immunological synapse forms at the interface between the two cells. It is still unclear how the cytoskeletal changes required for synapse formation are coordinated with signalling as a result of antigen recognition, but this study shows that the PDZ-domain-containing protein Discs large 1 (Dlg1) is involved. PDZ domains can support heterotypic and homotypic interactions, making proteins that contain them ideal scaffolding molecules. Indeed, Dlg1 is already known to be expressed at synapses between neurons in the central nervous system. In T cells, the authors show that Dlg1 is recruited with actin to the contact zone with an antigen-presenting cell, and it also associates with T-cell signalling molecules such as LCK. Dlg1 seems to inhibit signalling from the synapse: overexpression of the protein attenuated VAV1-induced transcription activity, whereas Dlg1 suppression potentiated the response to stimulation through CD3.

\section{PHAGOCYTOSIS}

Dectin-1 utilizes novel mechanisms for yeast phagocytosis in macrophages.

Herre, J. et al. Blood 10 August 2004 (doi:1182/blood-2004-03-1140).

The C-type lectin-like receptor Dectin-1 is required for the inflammatory response to $\beta$-glucans, such as those found in fungal pathogens. In this study, Dectin-1 is shown to mediate another process crucial for host defence - phagocytosis. Dectin-1-mediated internalization of the yeast particle zymosan required an ITAM-like motif in its cytoplasmic tail, similar to the ITAM sequence required for Fc $\gamma R$-mediated phagocytosis. However, Dectin-1-mediated uptake involved a different combination of signalling molecules than FcyR-mediated phagocytosis or than uptake mediated by other phagocytic receptors previously studied. Furthermore, when considering the biological importance of Dectin-1-mediated phagocytosis, it should be noted that its route of intracellular trafficking was dependent on the nature of the $\beta$-glucan ligand.

\title{
LYMPHOCYTE RESPONSES
}

\section{Linking arms: key role for IL-5}

Oxidative modification of low-density lipoprotein (LDL) present in atherosclerotic lesions induces both innate and adaptive immune responses, but until now, the relationship between these two arms of the immune response and their contributions to atherogenesis were unclear. Now, Binder et al. report that immunization with a model oxidized LDL - malondialdehyde-modified LDL (MDA-LDL) - promotes a T helper $2\left(\mathrm{~T}_{\mathrm{H}} 2\right)$-type response that, through the production of interleukin-5 (IL-5), increases the titre of natural antibodies specific for oxidized LDL. Moreover, the presence of these natural antibodies correlates with protection from atherosclerosis.

In a previous study, the authors showed that immunization of C57BL/6 mice with MDA-LDL induces a T-cell-dependent response and protects against atherosclerosis. After further analysis of this T-cell response, they found that it was of $\mathrm{T}_{\mathrm{H}} 2$ type - characterized by a predominance of IgG1 and the production of IL-5, IL-10 and IL-13 but not the $\mathrm{T}_{\mathrm{H}} 1$-type cytokine interferon- $\gamma$. The development of atherosclerosis in LDL receptor (LDLR)-deficient mice given a high-fat diet is associated with $\mathrm{T}_{\mathrm{H}} 1$-cell responses. However, when these mice were immunized with MDA-LDL, $\mathrm{T}_{\mathrm{H}}$ 2-cell responses dominated and the size of atherosclerotic lesions decreased compared with mice immunized with phosphate-buffered saline. The MDA-LDL-specific T cells produced high levels of IL-5 after in vitro stimulation with CD3- and CD28-specific antibodies, and MDALDL-immunized mice had markedly increased plasma levels of IL-5.

Surprisingly, they also found that immunization with MDA-LDL increased the titre of IgM that was specific for an epitope in oxidized LDL that was not present in the MDA-LDL inoculum - that is, the phosphorylcholine head group of oxidized phospholipids recognized by the natural IgM antibody known as T15/EO6. Natural antibodies are produced by $\mathrm{B} 1$ cells and are generally involved in T-cell-independent responses. So, how are B1 cells that produce the T15/EO6 antibody activated? Because IL-5 is produced at high levels following immunization with MDALDL, the authors tested whether this cytokine could augment the production of natural antibodies by B1 cells. Indeed, in vitro culturing of B1 cells with IL-5 promoted the secretion of T15/EO6 antibody, and intraperitoneal injections of IL-5 given to naive C57BL/ 6 mice increased T15/ EO6 titres. Moreover, although IL-5deficient mice and wild-type mice showed a similar bias to $\mathrm{T}_{\mathrm{H}} 2$-cell responses after immunization with MDA-LDL, IL-5-deficient mice had greatly reduced levels of T15/EO6 antibody.

To determine whether IL-5 is important in reducing atherosclerotic lesion size, the authors transplanted irradiated LDLR-deficient mice with bone marrow from IL-5-deficient or wild-type mice. Atherosclerosis was induced in these chimeric mice by feeding them a high-fat diet. Mice that were reconstituted with wild-type cells had smaller atherosclerotic lesions than those receiving IL-5-deficient cells, indicating that IL-5 has a protective role in atherogenesis, which is probably mediated through the secretion of natural antibodies by B1 cells. This is consistent with a previous observation that the T15/EO6 natural antibody can block the uptake of oxidized LDL by macrophages, which contribute to the pathogenesis of atherosclerotic lesions through the formation of lipid-loaded foam cells.

So, taken together, these data indicate that IL-5 provides a novel pathway linking the adaptive and natural arms of the immune system. This work has important implications for strategies that inhibit the actions of IL-5 to treat asthma and other allergic diseases.

Lucy Bird

(9) References and links ORIGINAL RESEARCH PAPER Binder et al. IL-5 links adaptive and natural immunity specific for epitopes of oxidized LDL and protects from atherosclerosis. J. Clin. Invest. 114, 427-437 (2004). 